\title{
A rare case of acute promyelocytic leukemia with IRF2BP2-RARA fusion; and literature review
}

This article was published in the following Dove Press journal: OncoTargets and Therapy

\author{
Yiping Liu (D* \\ Fang $\mathrm{Xu} \mathbb{1}^{*}$ \\ Hong $\mathrm{Hu}$ (D) \\ Jingjing Wen (D) \\ jing Su (D) \\ Qiaolin Zhou (D) \\ Wen Qu (D)
}

Department of Hematology, Mianyang Central Hospital, Mianyang 621000,

Sichuan, People's Republic of China

*These authors contributed equally to this work
Correspondence: Fang $\mathrm{Xu}$

Department of Hematology, Mianyang

Central Hospital, No. 12, Changjia Alley, Jingzhong Street, Fucheng District,

Mianyang 621000, People's Republic of China

Tel +86 | $377813882 \mid$

Fax +862157643271

Email147377807@qq.com
Background: Acute promyelocytic leukemia (APL) is commonly characterized by the fusion of retinoic acid receptor alpha (RARA) with promyelocytic leukemia (PML). Most APL patients acquire long-term survival after treatment with all-trans retinoic acid (ATRA) or arsenic agents-based chemotherapy.

Case presentation: A rare case of APL was reported after IRF2BP2-RARA was detected in the relapsed process using next-generation RNA-sequencing analysis. In addition, the mutation of NRAS was also detected. ATRA and arsenic trioxide combined with daunorubicin were used during induction treatment. The patient acquired complete remission but relapsed in 12 months. The patient was resistant to all other chemotherapies and refused any further therapy. The literature review indicated that allogeneic hematopoietic stem cell transplantation might be a therapeutic method to treat APL with IRF2BP2-RARA fusion.

Conclusion: Atypical APL should be considered even if the patients present with normal chromosomal karyotype and no classic PML-RARA fusions, but classical clinical features and bone marrow cell morphology. We reported a case of APL with IRF2BP2-RARA fusion was shown to harbor the NRAS mutation at relapse.

Keywords: acute promyelocytic leukemia, IRF2BP2-RARA, variant translocation, gene fusion, NRAS, mutation

\section{Background}

Acute promyelocytic leukemia (APL) is characterized by the fusion of retinoic acid receptor alpha (RARA) with promyelocytic leukemia (PML). In addition, about 2\% APL patients present variant fusions. ${ }^{1}$ Hitherto, $>10$ variant translocations have been reported to share the same C-terminal domains of RARA but different $\mathrm{N}$ terminal sequences. ${ }^{2}$ In 2015, Yin et al, for the first time, reported the occurrence of IRF2BP2-RARA. ${ }^{3}$ To date, four reports of IRF2BP2-RARA have been published worldwide. $^{3-6}$ Herein, we reported a case of relapse APL with IRF2BP2-RARA fusion from China.

\section{Case presentation}

A 32-year old woman was first hospitalized for menorrhagia and severe tiredness. Anemia with a hemoglobin of $64 \mathrm{~g} / \mathrm{L}$ and low platelet count of $33 \times 10^{9} / \mathrm{L}$ were noted. Blast cells were also observed in the peripheral blood smear. However, a normal activated partial prothrombin time (aPTT), fibrinogen level, and thrombin time were observed in addition to the prolonged prothrombin time (PT) of $14 \mathrm{~s}$. Her bone marrow was hypercellular with $57 \%$ promyelocytes, frequent Auer rods, and strong myeloperoxidase (Figure 1). All-trans retinoic acid (ATRA) and arsenic 


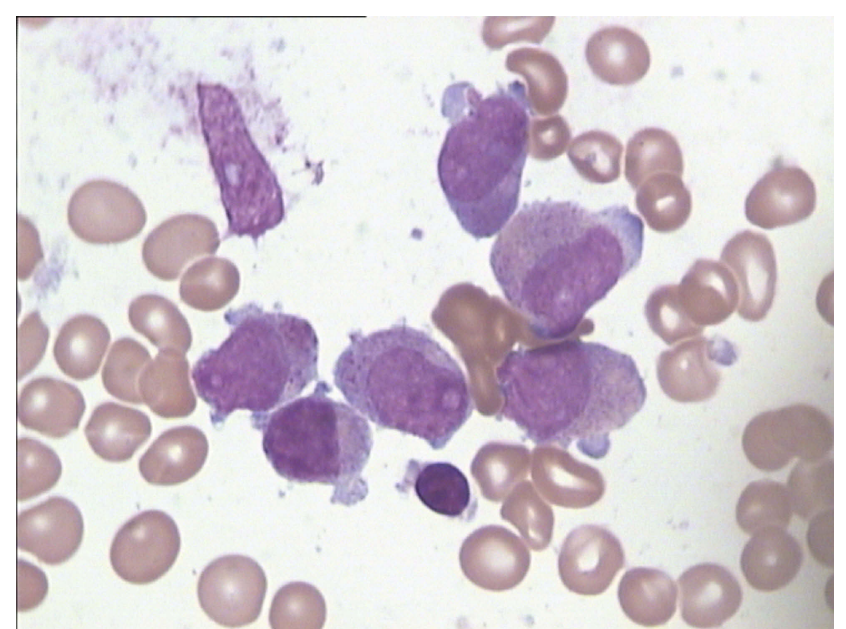

Figure I Bone marrow examination at diagnosis.

trioxide (ATO) were administered immediately after APL was suspected. Flow cytometry showed the immature cells expressing CD13, CD33, CD117, and CD64, and was negative for HLA-DR and CD34, CD3, CD4, CD56, CD5, CD10, CD19, CD20, CD7, CD11b, CD16, CD15,
CD14, CD36, CD41, and CD71. Chromosomal examination of leukemia cells revealed the karyotype 45, X, -X (Figure 2). Reverse transcription-polymerase chain reaction (RT-PCR) did not detect any translocations that were commonly detected in APL and other acute myelogenous leukemia including PML-RARA, PLZF-RARA, and NPM-RARA. Fluorescence in situ hybridization (FISH) failed to detect the fusion of RARA with PML or other gene partners. Also, no mutations were detected in FLT3, NPM1, CEBPA, WT1, or IDH1/2. Due to the inconsistency in morphology, immunological, cytogenetical, and molecular tests, the levels of PML-RARA, NPM-RARA, NuMA-RARA, FIPIL-RARA, PLZF-RARA, PPK-RARA, and STAT5b-RARA were examined for the second time by RT-PCR in the clinical laboratory of Huaxi Hospital. It was slightly positive for PML-RARA, thus leading to the diagnosis of APL.

After receiving ATRA (10 mg po. tid) and ATO (10 mg ivgtt qd) for 35 days, along with daunorubicin $\left(45 \mathrm{mg} / \mathrm{m}^{2}\right.$ on days 1-3), the patient acquired complete remission (CR). The consolidation chemotherapy comprised of treatment with

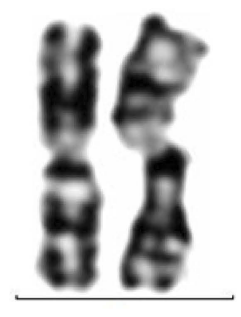

1

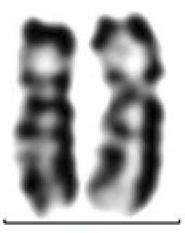

6

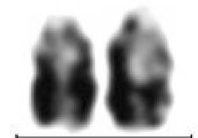

13

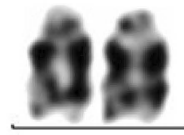

14

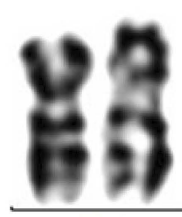

7

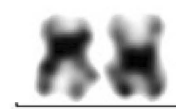

19

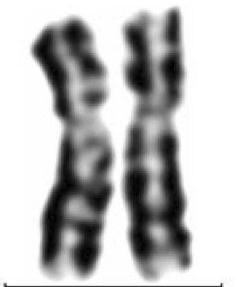

2

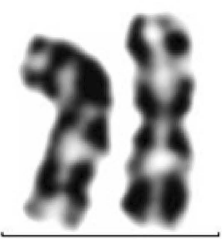

3

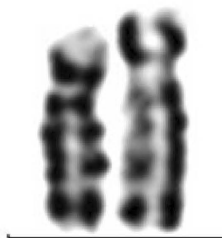

4

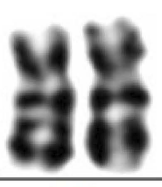

10

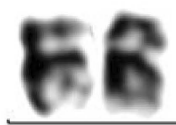

16

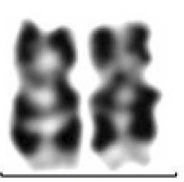

9

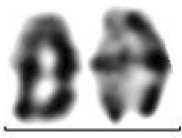

15

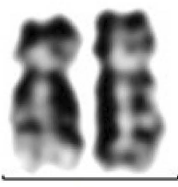

8

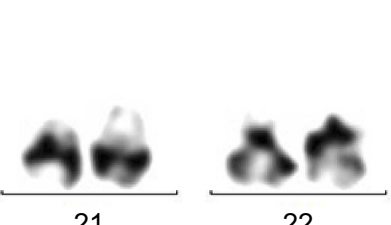

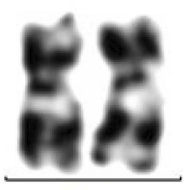

11

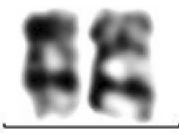

17

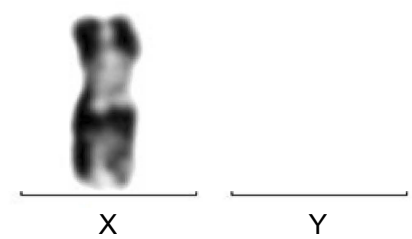

Figure 2 Chromosome karyotype analysis at first diagnosis: 45, X, -X [16]. 
DA (daunorubicin $45 \mathrm{mg} / \mathrm{m}^{2} /$ day on days 1-3; cytarabine $100 \mathrm{mg} / \mathrm{m}^{2} /$ day on days $1-7$ ), and HA (homoharringtonine $2 \mathrm{mg} / \mathrm{m}^{2} /$ day on days $1-5$, cytarabine $100 \mathrm{mg} / \mathrm{m}^{2} /$ day on days $1-7)$. ATRA (45 mg/m $/ \mathrm{m}^{2} \mathrm{~d}$ ) was administered on days 1-15 in combination with three single-agent chemotherapy courses. Subsequently, the patient who tested negative for PML/RARA was started on oral ATRA (10 mg tid) for 15 days, intravenous ATO (10 mg) for 15 days, and oral methotrexate (15 mg/m²/week) every 3 months. Methotrexate $(10 \mathrm{mg})$, cytarabine $(50 \mathrm{mg})$, and dexamethasone $(10 \mathrm{mg})$ were administered four times by spinal puncture to prevent the central nervous system leukemia. After 12 months, she presented with fever, blast cells were found in peripheral blood, and relapse was confirmed by bone marrow aspirate. RT-PCR and FISH still failed to detect the RARA arrangement. Cytogenetics revealed 45, X, -X [8]/45, idem, t $(9 ; 12)(q 13 ; p 11)[12]$, which differed from that during the initial diagnosis (Figure 3 ). The putative fusion gene was investigated by next-generation RNA-sequencing analysis. NRAS, BMP4 mutations, and the fusion between RARA and interferon regulatory factor 2 binding proteins 2 (IRF2BP2) were verified. This confirmed the variant case of APL. The patient received re-induction therapy, including ATRA, ATO, and daunorubicin; however, CR was not achieved. Then, CLAG protocol (cladribine $4 \mathrm{mg} / \mathrm{m}^{2}$ on days $1-5$; cytarabine $1000 \mathrm{mg} / \mathrm{m}^{2} \mathrm{q} 12 \mathrm{~h}$ on days $1-5$; G-CSF $300 \mu \mathrm{g}$ on days $\left.1-5\right)$ was administered. However, the patient still did not respond to the treatment and refused further therapy, and died eventually.

\section{Discussion}

APL is characterized by the fusion of RARA with PML or, rarely, other gene partners including PLZF, NPM, NuMA, STAT5b, PRKAR1A, FLP1L1, BCOR, OBFC2A, TBLR1, GTF2I, and STAT3. Herein, we reported a rare fusion IRF2P2-RARA detected in relapsed process. IRF2BP2 is localized at chromosome 1q42.3 and encodes a nuclear protein consisting of an $\mathrm{N}$-terminal zinc finger and a C-terminal RING finger domain that interacts specifically with the C-terminal transcriptional repression domain of IRF2. ${ }^{9}$ IRF2BP2 has also been involved in breast cancer cell lines, monoclonal gammopathy of undetermined significance, and other solid tumors. ${ }^{7,10,11}$

To date, four reports have been published on IRF2BP2RARA. $^{3-6}$ Clinical features of the APL with IRF2BP2-RARA were compared (Table 1). These 5 cases comprised of males
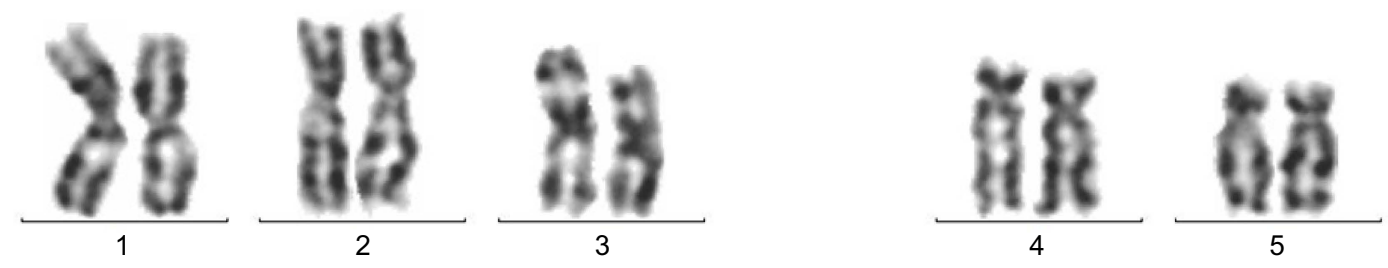

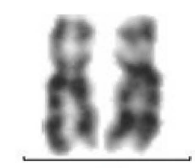

6

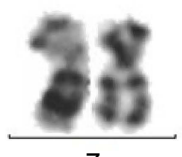

7

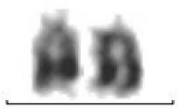

13

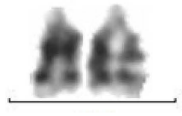

14

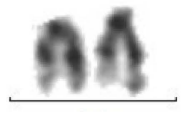

15
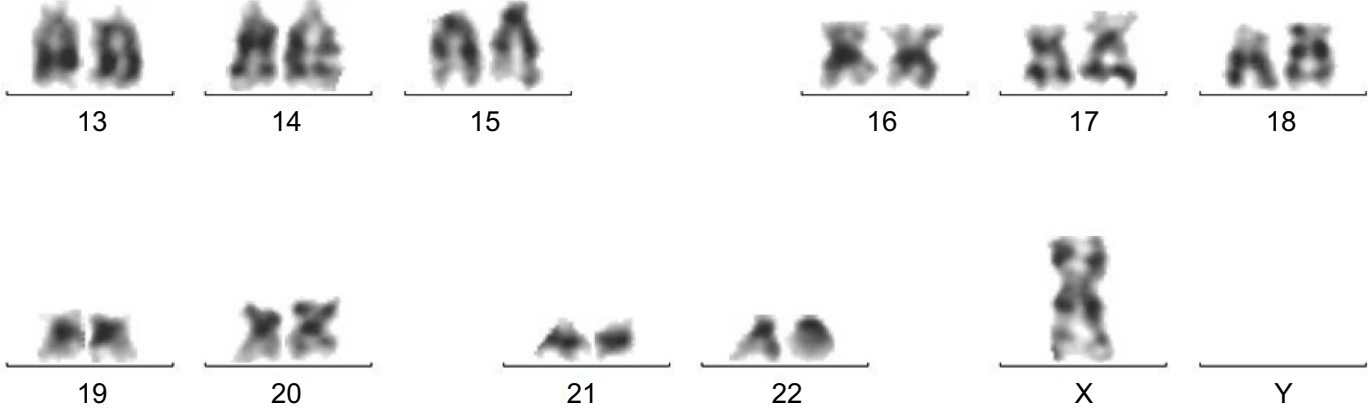

Figure 3 Chromosome karyotype analysis at relapse: 45, $X,-X[8] / 45$, idem, $t(9 ; \mid 2)(q|3 ; p| I)[I 2]$ 


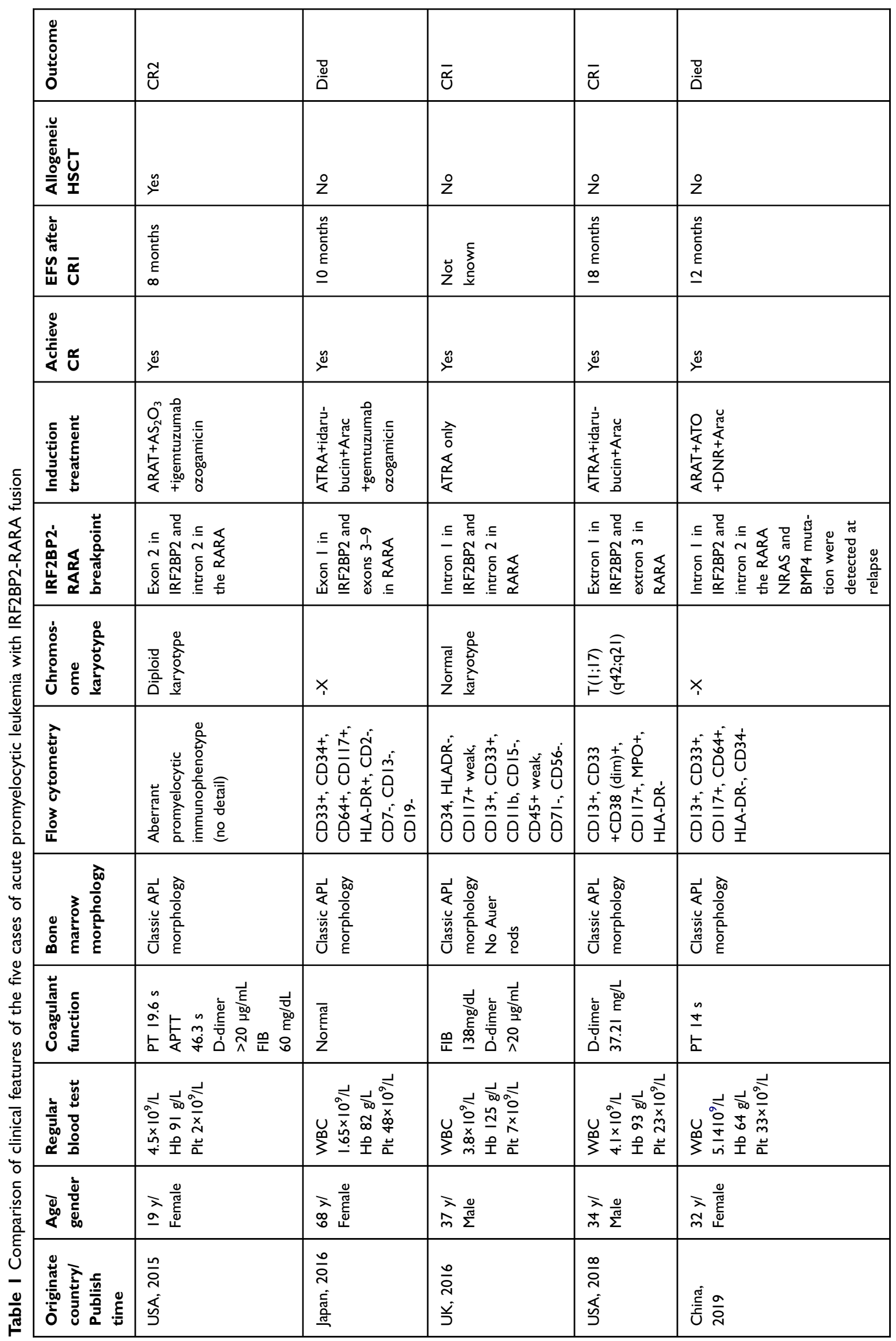


and females; 4 were $<40$-years-old. . All the patients presented different degrees of cytopenia or pancytopenia. Only two patients showed abnormal coagulant function with hypofibrinogenemia. Morphologically, four cases were identified as APL, except the case that was reported as atypical by Shimomura et al. ${ }^{3-6}$ Four cases exhibited classic immunophenotype of promyelocyte by flow cytometry (CD13, CD33, CD64, CD117-positive, and HLA-DR-negative), except one patient, who was HLA-DR-positive. The chromosome karyotype of $(1 ; 17)(\mathrm{q} 42 ; \mathrm{q} 21)$ was found in two cases, from which IRF2BP2-RARA was derived. In the present study, a case of a young female with cytopenia, mild abnormal coagulant function, classic morphology, and immunophenotype of APL at diagnosis was reported. Before IRF2BP2-RARA was verified, several examinations of the chromosome failed to find $(1 ; 17)$ (q42; q21), and normal karyotype was reported. All the cases including the current were stratified into low-intermediate risk. IRF2BP2-RARA was confirmed by next-generation RNAsequencing analysis. The breakpoint in the current case was at the same intron 2 in the RARA as reported by Yin et al in APL patients with IRF2BP2-RARA and respond differently to ATRA or ATO, which is usually inferior to typical APL. All the five cases were administered with ATRA at diagnosis, two combined with ATO, and three combined with anthracycline agents during the induction treatment. All cases acquired CR. The elderly patient from Japan failed to respond to ATRA, idarubicin, and cytarabine treatment. As reported, three patients relapsed and the event-free survival (RFS) was between 10 and 18 months.

Arsenic binds to the PML moiety of the PML-RARA fusion. ATRA binds to the RARA portion. Each drug initiates biochemically independent degradation pathways, ${ }^{12}$ which might partially explain the lack of response of APL with the variant fusions between RARA and other rare partners as compared to that of classic APL to arsenic agents due to the loss of binding sites. $^{2,8}$ In addition, variant fusions can inhibit the target of retinoic acid but are not sensitive to ATRA. This phenomenon might lead to ATRA to fail to inhibit the apoptosis pathway induced by RARA efficiently. ${ }^{13}$ In the four cases with IRF2BP2-RARA reported previously, all patients acquired CR after ATRA-based therapy, one received allotransplantation and acquired long term survival, three patients relapsed in 12 months, and two maintained CR before the article was published. Reportedly, the RARA fusions occur with other genes including PLZF, STAT5b, and GTF2I that are resistant to ATRA. ${ }^{13-16}$ Whether IRF2BP2 might be a regulatory factor to enhance the effect of RARA and cause resistance to ATRA is yet unknown.

NRAS mutation was found in the patient in this study and the 19-year-old female from the USA. EFS after the first CR was 10 and 12 months, respectively. NRAS mutation was common in myeloid tumors, lymphoma, lymphocyte leukemia, melanoma, intestinal cancer, lung cancer, and pancreatic cancer. ${ }^{17-19}$ Furthermore, NRAS mutation was found in $14.7 \%$ of acute myeloid leukemia patients. ${ }^{20}$ Welch et al compared the most mutations in acute myeloid leukemia and APL. The research concluded that in many cases, only one or two additional, cooperating mutations were essential to generate the malignant founding clone. The cells from the founding clone can acquire additional cooperating mutations, yielding subclones that contribute to disease progression and/or relapse. ${ }^{21}$ Madan et al explored the mutational landscape using whole-exome $(n=12)$ and subsequent targeted sequencing of 398 genes in 153 primary and 69 relapse APLs. Also, the recurrent alterations in FLT3, WT1, NRAS, and KRAS mutation were observed in the newly diagnosed APL, whereas mutations in the other genes commonly mutated in myeloid leukemia were rarely detected. NRAS mutation occurred at a higher frequency in newly diagnosed samples as compared to with the relapse $(9.7 \%$ in newly diagnosed and $5.2 \%$ in relapse). ${ }^{22}$ Thus, these studies might explain the poor prognosis of the APL patient with IRF2BP2-RARA and NRAS mutation. However, the combined effects of IRF2BP2-RARA and NRAS mutation in APL need to be investigated further.

It is worthy of note that only 2 cases of APL with IRF2BP2-RARA presented with hypofibrinogenemia (40\%). The incidence of abnormal coagulant function seemed less than typical APL. Wang $X$ et al reviewed characteristics of STAT5b/RARa APL patients. 9 in 12 cases were diagnosed as disseminated intravascular coagulation. Whether the lower incidence of abnormal coagulant function was the clinical feature of APL with IRF2BP2-RARA, further observation is needed. ${ }^{23}$

\section{Conclusion}

Patients were suspected to exhibited APL based on the clinical features and bone marrow cell morphology. The abnormal chromosomal karyotype and classic PML-RARA fusion test were not sufficient to exclude APL. Thus, variant APL should be considered. IRF2BP2-RARA is a rare variant fusion in APL, and only five cases were reported worldwide. ATRA and arsenic agent-based induction treatment are still 
effective for APL with IRF2BP2-RARA fusion. However, allogeneic hematopoietic stem cell transplantation might be a therapeutic method to acquire long-term survival.

\section{Ethics approval and informed consent}

The study protocol was approved by the Ethics Committees of the Mianyang Central Hospital (P2019003), and the participant provided written informed consent.

\section{Consent for publication}

Informed written consent was obtained from the patient for publication of this case report and accompanying images.

\section{Data availability}

The datasets used and/or analyzed during the current study are available from the corresponding author on reasonable request

\section{Abbreviations}

APL, Acute promyelocytic leukemia; RARA, retinoic acid receptor alpha; ATRA, all-trans retinoic acid; PML, promyelocytic leukemia; aPTT, activated partial prothrombin time; PT, prothrombin time; ATO, arsenic trioxide.

\section{Author contributions}

All authors contributed to data analysis, drafting or revising the article, gave final approval of the version to be published, and agree to be accountable for all aspects of the work.

\section{Disclosure}

The authors report no conflicts of interest in this work.

\section{References}

1. Hematology CSo, Association CMD. [Chinese guidelines for diagnosis and treatment of acute promyelocytic leukemia (2018)]. Zhonghua Хие Yе Xие Za Zhi. 2018;39(3):179-183. doi:10.3760/cma.j.issn.02532727.2018.03.002

2. Yan W, Molecular Characteristics ZG. Clinical significance of 12 fusion genes in acute promyelocytic leukemia: a systematic review. Acta Haematol. 2016;136(1):1-15. doi:10.1159/000444514

3. Yin CC, Jain N, Mehrotra M, et al. Identification of a novel fusion gene, IRF2BP2-RARA, in acute promyelocytic leukemia. $J$ Natl Compr Canc Netw. 2015;13(1):19-22.

4. Shimomura Y, Mitsui H, Yamashita Y, et al. New variant of acute promyelocytic leukemia with IRF2BP2-RARA fusion. Cancer Sci. 2016;107(8):1165-1168. doi:10.1111/cas.12970
5. Jovanovic JV, Chillon MC, Vincent-Fabert C, et al. The cryptic IRF2BP2-RARA fusion transforms hematopoietic stem/progenitor cells and induces retinoid-sensitive acute promyelocytic leukemia. Leukemia. 2017;31(3):747-751. doi:10.1038/leu.2016.338

6. Mazharuddin S, Chattopadhyay A, Levy MY, Redner RL. IRF2BP2RARA $\mathrm{t}(1 ; 17)(\mathrm{q} 42.3 ; \mathrm{q} 21.2)$ APL blasts differentiate in response to all-trans retinoic acid. Leuk Lymphoma. 2018;59(9):2246-2249. doi:10.1080/10428194.2017.1421761

7. Soliman H, Khalil F, Antonia S. PD-L1 expression is increased in a subset of basal type breast cancer cells. PLoS One. 2014;9(2):e88557. doi:10.1371/journal.pone.0088557

8. Yao L, Wen L, Wang N, et al. Identification of novel recurrent STAT3-RARA fusions in acute promyelocytic leukemia lacking $\mathrm{t}$ (15;17)(q22;q12)/PML-RARA. Blood. 2018;131(8):935-939. doi:10.1182/blood-2017-09-807370

9. Carneiro FR, Ramalho-Oliveira R, Mognol GP, Viola JP. Interferon regulatory factor 2 binding protein 2 is a new NFAT1 partner and represses its transcriptional activity. Mol Cell Biol. 2011;31 (14):2889-2901. doi:10.1128/MCB.00974-10

10. Blotta S, Tassone P, Prabhala RH, et al. Identification of novel antigens with induced immune response in monoclonal gammopathy of undetermined significance. Blood. 2009;114(15):3276-3284. doi:10.1182/blood-2009-04-219436

11. Nyquist KB, Panagopoulos I, Thorsen J, et al. Whole-transcriptome sequencing identifies novel IRF2BP2-CDX1 fusion gene brought about by translocation $\mathrm{t}(1 ; 5)(\mathrm{q} 42 ; \mathrm{q} 32)$ in mesenchymal chondrosarcoma. PLoS One. 2012;7(11):e49705. doi:10.1371/journal.pone. 0049705

12. Alice $\mathrm{V}$, Jehannine A. Resistance to therapy in acute promyelocytic leukemia. $N$ Engl J Med. 2014;371(12):1170-1172. doi:10.1056/ NEJMc1409040

13. He LZ, Guidez F, Tribioli C, et al. Distinct interactions of PMLRARalpha and PLZF-RARalpha with co-repressors determine differential responses to RA in APL. Nat Genet. 1998;18(2):126-135. doi:10.1038/ng0298-126

14. Licht JD, Chomienne C, Goy A, et al. Clinical and molecular characterization of a rare syndrome of acute promyelocytic leukemia associated with translocation (11;17). Blood. 1995;85 (4):1083-1094.

15. Li J, Zhong HY, Zhang Y, et al. GTF2I-RARA is a novel fusion transcript in a $\mathrm{t}(7 ; 17)$ variant of acute promyelocytic leukaemia with clinical resistance to retinoic acid. Br J Haematol. 2015;168(6):904908. doi:10.1111/bjh. 13157

16. Dong S, Tweardy DJ. Interactions of STAT5b-RARalpha, a novel acute promyelocytic leukemia fusion protein, with retinoic acid receptor and STAT3 signaling pathways. Blood. 2002;99(8):26372646. doi:10.1182/blood.v99.8.2637

17. Burd CE, Liu W, Huynh MV, et al. Mutation-specific RAS oncogenicity explains NRAS codon 61 selection in melanoma. Cancer Discov. 2014;4(12):1418-1429. doi:10.1158/2159-8290.CD-14-0729

18. Molnar B, Galamb O, Peterfia B, et al. Gene promoter and exon DNA methylation changes in colon cancer development - mRNA expression and tumor mutation alterations. BMC Cancer. 2018;18(1):695. doi:10.1186/s12885-018-4242-8

19. Jakobsen JN, Santoni-Rugiu E, Grauslund M, Melchior L, Sorensen JB. Concomitant driver mutations in advanced EGFRmutated non-small-cell lung cancer and their impact on erlotinib treatment. Oncotarget. 2018;9(40):26195-26208. doi:10.18632/ oncotarget. 25490

20. Borthakur G, Popplewell L, Boyiadzis M, et al. Activity of the oral mitogen-activated protein kinase kinase inhibitor trametinib in RASmutant relapsed or refractory myeloid malignancies. Cancer. 2016;122(12):1871-1879. doi:10.1002/cncr.29986 
21. Kirchberger MC, Ugurel S, Mangana J, et al. MEK inhibition may increase survival of NRAS-mutated melanoma patients treated with checkpoint blockade: results of a retrospective multicentre analysis of 364 patients. Eur J Cancer. 2018;98:10-16. doi:10.1016/j. ejca.2018.04.010

22. Zhang Y, Wang F, Chen X, et al. Mutation profiling of 16 candidate genes in de novo acute myeloid leukemia patients. Front Med. 2019;13(2):229-237. doi:10.1007/s 11684-018-0616-1
23. Wang X, Wang J, Zhang L. Characterization of atypical acute promyelocytic leukaemia: three cases report and literature review. Medicine (Baltimore). 2019;98(19):e15537. doi:10.1097/MD.00 00000000015537

\section{Publish your work in this journal}

OncoTargets and Therapy is an international, peer-reviewed, open access journal focusing on the pathological basis of all cancers, potential targets for therapy and treatment protocols employed to improve the management of cancer patients. The journal also focuses on the impact of management programs and new therapeutic

Submit your manuscript here: https://www.dovepress.com/oncotargets-and-therapy-journal agents and protocols on patient perspectives such as quality of life, adherence and satisfaction. The manuscript management system is completely online and includes a very quick and fair peer-review system, which is all easy to use. Visit http://www.dovepress.com/ testimonials.php to read real quotes from published authors. 